\title{
Les lâchers morphogènes depuis un barrage justification opérationnelle et protocole d'intervention
}

\author{
Rémi Loire ${ }^{1}$, Hervé Piégay ${ }^{2}$, Jean-René Malavoi ${ }^{1, *}$, G. Mathias Kondolf ${ }^{3}$ et Gabriel Melun ${ }^{4}$ \\ ${ }^{1}$ EDF HYDRO, Centre d'ingénierie hydraulique-Savoie Technolac, 4, allée du Lac de Tignes, 73290 La Motte-Servolex, France \\ ${ }^{2}$ Université de Lyon, CNRS UMR5600, Site ENS, 15, Parvis R.-Descartes, 69007 Lyon, France \\ ${ }^{3}$ University of California Berkeley, Department of Landscape Architecture and Environmental Planning,202 Wurster Hall, Berkeley, \\ États-Unis \\ ${ }^{4}$ Office français de la biodiversité, Direction de la recherche et de l'appui scientifique, 5, allée Felix-Nadar, 94300 Vincennes, France
}

Reçu le 13 décembre 2019 / Accepté le 1 juillet 2020

Résumé - Nous proposons une nouvelle approche méthodologique pour évaluer la pertinence et mettre en œuvre des « lâchers morphogènes » en aval de barrages hydroélectriques. Ces lâchers correspondent à des débits relativement élevés lâchés par les barrages afin de générer des changements dans les caractéristiques physiques des lits fluviaux (par exemple, décolmatage, désensablement, élargissement des bandes actives alluviales, augmentation de la charge sédimentaire grossière, gestion de la végétation riveraine...) et de créer ou d'améliorer les habitats aquatiques et riverains. La méthodologie proposée repose sur une analyse de la littérature scientifique et sur nos propres expériences. Nous introduisons quelques méthodes de dimensionnement de ces lâchers (intensité, durée, forme de l'hydrogramme, variation saisonnière et récurrence), et évaluons les conflits potentiels avec divers enjeux environnementaux, socioéconomiques et opérationnels associés aux barrages. Nous proposons également des solutions alternatives lorsque ces lâchers ne peuvent être mis en œuvre, du fait notamment de contraintes trop lourdes. La procédure d'évaluation et de mise en œuvre des lâchers morphogènes se veut rigoureuse, itérative et basée sur plusieurs étapes, dont la plupart doivent être validées par les diverses parties prenantes : un diagnostic préliminaire quantifiable, la définition d'objectifs clairs, la prise en compte des contraintes présentes sur les sites, le dimensionnement du lâcher et sa mise en œuvre. Une phase d'évaluation est également proposée pour tirer des leçons des expériences et améliorer les actions.

Mots clés : débit écologique / débit environnemental / lâcher morphogène / débit de maintenance / barrage / gestion adaptative

Abstract - Morphogenic releases: Why? How? We propose a new methodological approach for studying
and implementing morphogenic flow releases downstream of hydroelectric dams. These morphogenic flows
are high flows released from the dams and designed to produce changes in physical characters of channel
features (e.g., grain size fining, prevention of clogging, sand export, channel widening, bedload transport
increase...) and to create or improve aquatic and riparian habitats. We compile approaches from scientific
literature and from our own experiments, to specify the dimensions of these flow releases (magnitude,
duration, shape of hydrograph, seasonal variation, and recurrence), and assess potential conflicts with
environmental, socio-economic and dam-operational issues. We also propose alternative actions when such
flows cannot be implemented. The process of studying and implementing morphogenic flow releases is
based on several stages: a quantifiable preliminary diagnosis, the definition of clear objectives, addressing
constraints present at the sites, dimensioning of the release and implementation of the release. A phase of
assessment, usually based on monitoring is also proposed to learn from experiences and improve actions.

Keywords: environmental flow / flushing flow / maintenance flow / eflood / dam / adaptive management

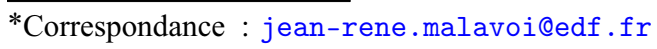




\section{Introduction}

Les impacts des barrages sur le système fluvial aval sont aujourd'hui bien documentés (Williams et Wolman, 1984; Kondolf, 1997; Brandt, 2000; Petts et Gurnell, 2005, entre autres). Pour ce qui concerne leurs impacts hydrologiques, ces auteurs notent une influence plus ou moins prononcée sur le régime des crues (débit, pic, fréquence, durée), ce qui entraîne de facto une modification de l'occurrence des «débits morphogènes » naturels dans le tronçon situé en aval (voir plus loin). Parmi les principaux impacts géomorphologiques recensés, plusieurs auteurs (Kondolf, 1997; Brandt, 2000; Petts et Gurnell, 2005) ont notamment constaté une altération des processus sédimentaires, tels que des dépôts importants de sédiments fins ou grossiers, ou, au contraire, l'absence totale de transport solide, le manque de renouvellement des formes fluviales ou encore un développement exacerbé de la végétation terrestre ou aquatique. Ces altérations géomorphologiques peuvent elles-mêmes engendrer différents types d'impacts: des impacts «écologiques» au sens large, en entraînant notamment des altérations de la structure et/ou de la disponibilité des habitats aquatiques et rivulaires indispensables au bon fonctionnement des biocénoses (Arthington et Zalucki, 1998), voire des impacts «sociétaux » en affectant les biens et les personnes, du fait par exemple de l'augmentation de la fréquence des inondations sous l'effet de dépôts sédimentaires excessifs ou du trop grand développement de la végétation alluviale dans le lit mineur.

Face à ces différents impacts et pour tenter de les résorber, de nombreux chercheurs et gestionnaires de cours d'eau, ont réalisé différentes expérimentations au cours des dernières décennies. La plupart d'entre elles se concentraient sur l'un des compartiments les plus affectés par la mise en service de barrages, à savoir l'hydrologie (Konrad et al., 2011; Olden et al., 2014). Parmi ces expérimentations, beaucoup concernaient le lâcher de débits élevés, potentiellement «morphogènes », afin de générer la mobilité des sédiments et l'évolution des formes fluviales.

\section{Les concepts hydrauliques et geomorphologiques «classiques»: debit critique - debit morphogene - debit dominant - debit de pleins bords, etc.}

Débit critique, débit morphogène, débit dominant, débit de pleins bords, etc. ces différents termes sont des concepts de base de la géomorphologie fluviale. Ils ont été pour la plupart développés depuis les années 1950 dans le contexte de cours d'eau «naturels ». La notion de débit «morphogène» n'existe pas dans la littérature anglo-saxonne. Il vient tout juste d'être proposé (Loire et al., sous presse). Deux approches scientifiques ont conduit à aborder la question de débits qui pourraient agir sur les formes fluviales et être donc morphogènes, une approche dite de "géométrie hydraulique» et une approche classique de mesure du transport solide.

\subsection{La géométrie hydraulique}

Cette approche émerge à la fin des années 1950 considérant qu'il est possible de prédire la géométrie d'un chenal, principalement sa profondeur et sa largeur à partir d'une valeur de débit (Wolman et Leopold, 1957; Leopold et al., 1964; Williams, 1978...). De tels travaux sont toujours explorés (voir par exemple l'article récent de Morel et al., 2019).

Dans ce cadre, on distingue le débit à pleins bords considéré en France comme le débit morphogène qui est à l'origine en définitive de la taille du lit. Depuis la publication de Wolman et Leopold (1957), on considère que ce débit à pleins bords et morphogène correspond à un débit dominant $(\mathrm{Q} 1.3$ an ou $\mathrm{Q} 1.5)$... d'où la confusion qui est parfois fait entre ces termes. Le débit à pleins bords est un débit dominant lorsque la géométrie fluviale n'est pas perturbée par des pressions humaines, d'où tout le travail qui est conduit depuis 1957 pour identifier ainsi les géométries naturelles et les géométries perturbées. Ces travaux s'appliquent bien à des rivières à lit unique et plutôt à faible transport solide où le vecteur morphogène est lié au débit liquide. Lorsque le transport solide devient important, il s'applique beaucoup moins. Certains auteurs ont ainsi montré que le débit dominant n'occupe qu'une petite partie du corridor graveleux d'une rivière en tresses et que le renouvellement d'une tresse avec l'érosion de la végétation pionnière est avant tout régi par les crues décennales.

\subsection{La mesure du transport solide}

Le débit le plus classique dans ce cadre correspond au débit de début de mouvement ou débit critique. C'est le débit à partir duquel on commence à observer les premières mises en mouvement des particules élémentaires du substrat alluvial dans le fond du lit mineur d'un cours d'eau. De nombreux auteurs ont pu établir les valeurs de ce type de débit grâce à diverses méthodes de mesures in situ telles que la peinture de particules et les marquages divers (pit tags), les hydrophones, etc. (Milhous et Klingeman, 1973; Helland-Hansen et al., 1974; Klingeman et al., 1994). Il est aussi possible de calculer ce débit sur des bases théoriques, mais avec une forte marge d'incertitude (Hjulström, 1935; Shields, 1936; etc.).

La valeur de ce débit de début de mouvement est très variable selon les cours d'eau mais une dichotomie très nette existe entre les rivières à sable (où il peut être très bas en fréquence, en tout cas bien inférieur au module) et les rivières à gravier (où il est plutôt situé au-dessus, voire très au-dessus du module).

Ce débit constitue ainsi un seuil au-delà duquel le débit devient alors significativement morphogène dans la mesure où il mobilisera alors une partie de plus en plus significative des alluvions grossières disponibles dans le lit.

Le débit dominant ou efficace est souvent défini comme le débit le plus efficace en termes de transport de sédiments et de «travail» morphogénique au sens physique (Wolman et Miller, 1960; Benson et Thomas, 1966). D'un point de vue théorique, ce débit est classiquement obtenu en évaluant pour chacun des débits d'une courbe de débits classés le transport solide (via une formule semi-empirique ou une courbe de tarage hydro-sédimentaire). En faisant le produit entre le flux solide et l'occurrence, on peut retrouver un pic de transport solide (débit dominant) (Camenen et al., 2015).

\subsection{Remarques générales}

Il est important de noter qu'un même débit peut se traduire par une activité morphogénique plus ou moins forte selon l'intensité et la fréquence des évènements hydrologiques 


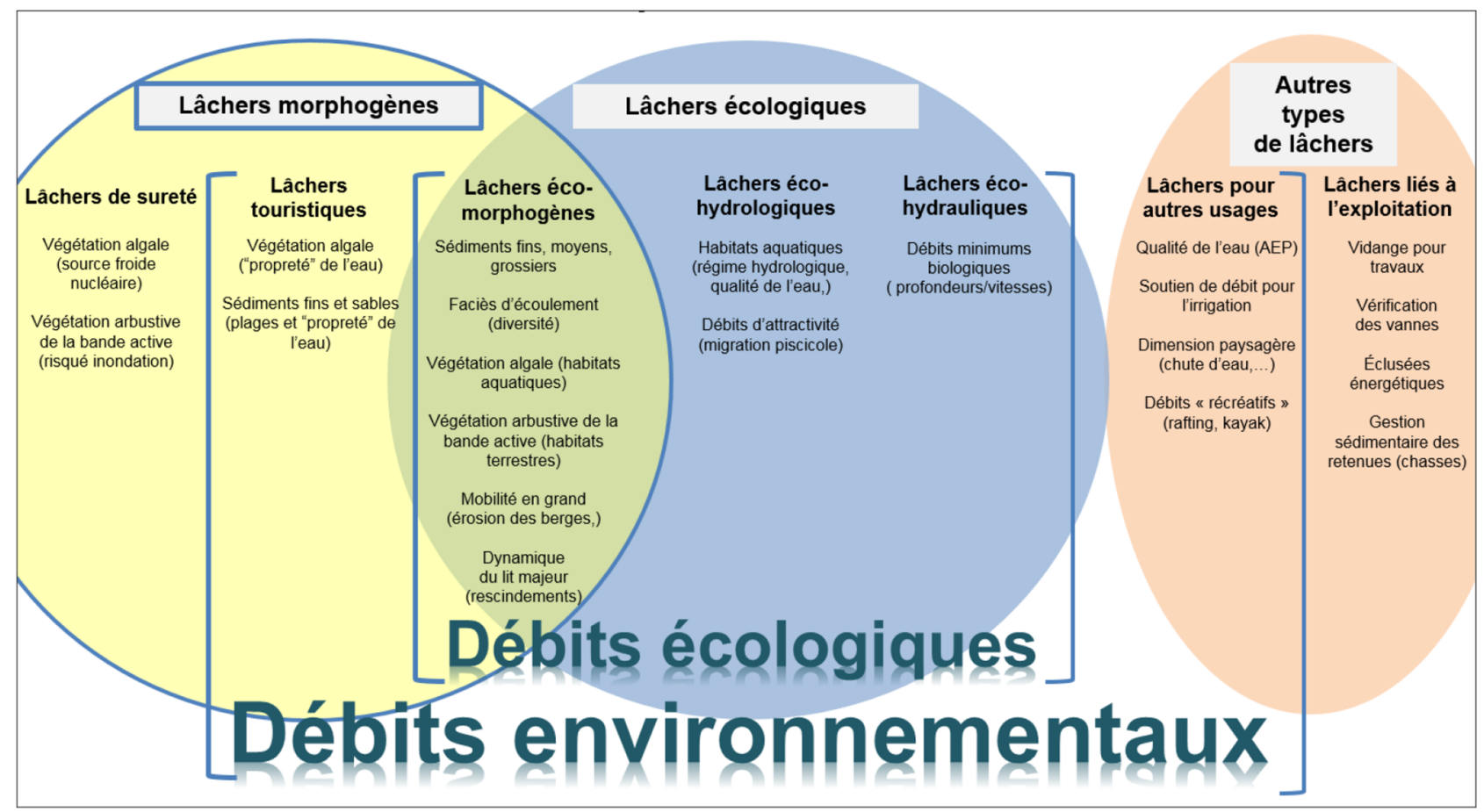

Fig. 1. Types de lâchers d'eau à l'aval d'un barrage et objectifs visés.

survenus lors des mois ou des années précédentes. Par exemple, un débit de $n \mathrm{~m}^{3} / \mathrm{s}$ survenant après cinq ans de faible hydraulicité aura une moindre efficacité morphogénique que le même débit survenant un an après une crue décennale ayant arraché toute la végétation alluviale, rompu l'armure de surface et érodé des kilomètres de berges sablo-graveleuses.

Il faut souligner aussi que la durée de l'évènement est fondamentale. Un jour de débit $n$ sera probablement moins «morphogène » que cinq jours de débit 0,7 ou $0,8 n$.

Toute la question en fait est de définir ce que veut dire morphogène? La création et le maintien d'une géométrie de chenal, la mise en mouvement du fond... ou plus modestement le transit d'un flux sableux sur un fond non mobile... Tout est question d'échelle et dans le cadre de la mise en place de lâchers ayant pour objectif d'induire un effet morphologique et d'être ainsi morphogène, il y a un gradient possible d'actions.

\section{Les concepts de gestion axes sur des lâchers d'eau volontaires: des débits morphogènes aux lâchers morphogènes}

\subsection{Le contexte actuel}

Dans ce cadre, il est important de distinguer explicitement «lâcher morphogène » et « débit morphogène », le lâcher étant une action volontaire réalisée par le gestionnaire d'un barrage pour répondre à certains objectifs qu'ils soient d'ordres écologiques, liés à l'exercice de certains usages ou encore à la sûreté et au maintien de l'exploitation de l'ouvrage (Fig. 1). Ce peut être aussi une action volontaire de non-entonnement et déversement d'un débit qui devrait théoriquement être turbiné ou stocké.

\subsubsection{Les différents types de lâchers d'eau depuis un barrage}

Le schéma ci-dessous présente de manière quasi exhaustive, les différents types de lâchers d'eau qu'un gestionnaire de barrage, hydroélectrique ou non, peut être amené à réaliser volontairement, pour satisfaire différents objectifs. Nous les avons replacés dans le champ sémantique couvert par deux concepts qui ont émergé récemment: les débits écologiques (ecological flows) et les débits environnementaux (environmental flows), les deux étant souvent regroupés sous le terme générique d'e-flows en anglais qui n'a pas vraiment d'équivalent en français et qui justifient la présentation détaillée de ces éléments.

Les débits écologiques, particulièrement en Europe dans le cadre de la mise en œuvre de la DCE, désignent la quantité d'eau et le régime hydrologique, nécessaire à la préservation de la diversité des biocénoses aquatiques et des fonctionnalités de l'hydrosystème. Les débits environnementaux intègrent les précédents et s'étendent à des objectifs plus larges tels que la qualité paysagère, les loisirs liés à l'eau, etc.

On distingue deux grands types d'objectifs des lâchers: morphogènes et écologiques, les deux se recoupant autour de l'objet principal de cette contribution. Une troisième catégorie regroupe encore d'autres objectifs.

\subsubsection{Lâchers morphogènes sensu stricto}

Certains lâchers morphogènes sensu stricto sont réalisés pour assurer des objectifs non strictement «écologiques ».

\section{Les lâchers de sûreté}

Les lâchers de sûreté induisent des effets biogéomorphologiques destinées à assurer la sûreté des populations. Un 
exemple récent est le programme de lâchers morphogènes visant la réduction des importants volumes d'algues macrophytes présentes sur la rivière Ebre en Espagne, volumes qui empêchent notamment la bonne alimentation en eau froide des centrales nucléaires présentes le long du cours d'eau (Batalla et al., 2014). Certains lâchers sont aussi préconisés pour limiter le développement végétal dans des sections en débit réservé afin d'assurer la débitance du chenal en période de crue.

\section{Les lâchers à des fins touristiques}

Des lâchers morphogènes peuvent également être réalisés pour des besoins touristiques. Par exemple, sur le Colorado (Powell, 2002), même si les lâchers réalisés ont plusieurs objectifs, l'un des principaux est de permettre la re-création de bancs de sable disparus depuis des années suite à la construction de barrages. Les lâchers doivent favoriser hydrauliquement la remise en suspension des sables apportés par les affluents et leur redépôt sur les parties hautes des bancs, pour permettre aux touristes de bénéficier de plages lors de leur descente en raft.

\subsubsection{Lâchers écologiques sensu stricto}

Certains types de lâchers, sans vocation morphogène, visent directement l'amélioration du fonctionnement écologique des cours d'eau.

\section{Les lâchers éco-hydrauliques}

Depuis plusieurs décennies déjà, des débits minimums biologiques sont délivrés par les barrages sur les tronçons de rivière court-circuités par des dérivations hydroélectriques. Ils visent ainsi à modifier les conditions hydrauliques du chenal afin de s'approcher des préférendums d'habitat pour différences espèces de poissons, principalement en termes de hauteur d'eau et de vitesse.

\section{Les lâchers éco-hydrologiques}

C'est à partir de la fin des années 1990 que l'idée d'un régime hydrologique a progressivement vu le jour, dans l'objectif d'améliorer les processus écologiques dans la rivière et sur ses marges, ainsi que les habitats aquatiques dans leur ensemble. Le principe est de se rapprocher des gammes de débits naturels, de l'étiage aux évènements de crue en passant par la mise en œuvre de «débits d'appel» favorisant la montaison des poissons et d'une certaine saisonnalité.

\section{Les lâchers écomorphogènes}

Certains types de lâchers morphogènes, en forte augmentation au cours des dernières décennies, visent à améliorer la qualité globale des milieux aquatiques. Ceux visant l'amélioration de la reproduction piscicole ou la restauration des habitats aquatiques sont de loin les plus nombreux. Ils consistent à " délimoner» ou dessabler le substrat graveleux des zones de reproduction ou de grossissement de certaines espèces de poissons. Petit à petit ont été proposés d'autres objectifs tels que l'amélioration des habitats via la réactivation de processus géomorphologiques : érosion des berges, reprise des sédiments apportés par les affluents, maîtrise du développement de la végétation aquatique ou rivulaire. Enfin, certains lâchers, de plus forte intensité encore, sont parfois réalisés pour restaurer des zones humides en lit majeur, voire certaines fonctionnalités de la forêt alluviale.

Nous appellerons donc « lâchers écomorphogènes » reprenant ainsi les termes de Loire et al, (sous presse) les lâchers morphogènes à objectifs principalement écologiques.

\subsubsection{Des lâchers répondant à des objectifs d'usage ou d'exploitation de l'ouvrage}

Certains types de lâcher d'eau sont parfois réalisés pour répondre à d'autres objectifs.

\section{Les lâchers destinés à assurer certains usages}

On y trouve des opérations assez «classiques» telles que des lâchers pour améliorer la qualité de l'eau en aval (pour l'eau potable notamment), soutenir l'irrigation, conserver assez d'eau sur une chute d'eau naturelle (dimension paysagère), fournir des conditions de débit permettant la pratique des sports d'eau vive, etc.

\section{Les lâchers liés à la bonne exploitation des barrages}

Ces lâchers d'eau concernent la gestion intrinsèque des barrages. On distingue par exemple des lâchers préalables à des opérations de maintenance des organes de contrôle (on parle alors généralement de vidanges), des vidanges obligatoires pour les inspections, des lâchers énergétiques (éclusées).

La plupart du temps, ces lâchers ne sont pas morphogènes dans la mesure où ils n'affectent pas la morphologie du lit ou son substrat mais il arrive que certains le soient, sans intention initiale (les lâchers pour les sports d'eau vive notamment), génèrent parfois des vitesses critiques qui mobilisent certaines classes granulométriques du substrat alluvial. Se rangent également dans cette catégorie, les chasses qui visent à limiter la sédimentation dans les retenues et qui peuvent être morphogènes du fait des effets induits dans le réservoir et des effets potentiels aval, résultant par exemple du colmatage intersticiel.

\subsubsection{Périmètre et objectifs des lâchers d'eau morphogènes}

Si l'on s'en réfère aux dernières données fournies par la France dans le cadre de la mise en œuvre de la DCE (2015), plus de $55 \%$ des masses d'eau de surface sont dans un état écologique dégradé ou très dégradé. Pour un grand nombre de ces masses d'eau de surface, le principal obstacle au bon état écologique est un problème de dysfonctionnement hydromorphologique et donc de qualité des habitats aquatiques et rivulaires.

Rappelons qu'un bon fonctionnement hydromorphologique peut être caractérisé par une grande diversité de faciès d'écoulement, des berges naturelles, des bancs alluviaux mobiles, une ripisylve variée, des annexes hydrauliques fonctionnelles et, surtout, une dynamique fluviale peut ou pas entravée. La dynamique fluviale est en effet le moteur de la création et de la régénération d'une diversité d'habitats aquatiques et rivulaires indispensable aux biocénoses fluviatiles.

C'est ainsi le concept d'habitat notamment, qui lie les compartiments «hydromorphologiques» et «biologiques». 


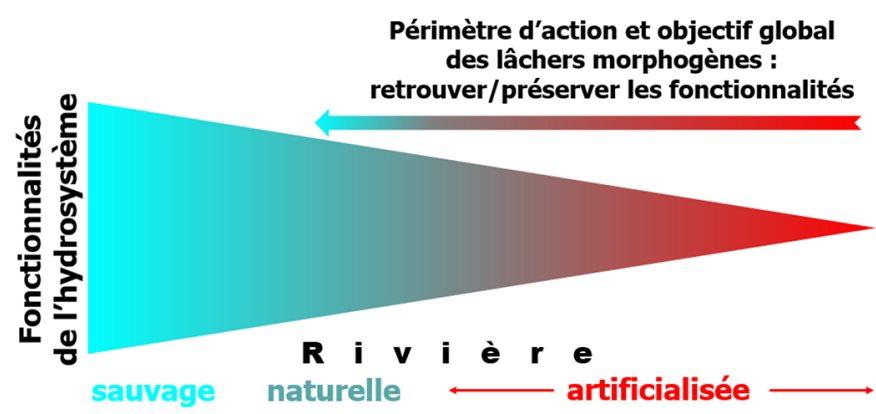

Fig. 2. Schéma conceptuel du périmètre d'action des lâchers morphogènes.

L'hydrologie, et particulièrement le régime des crues, est l'un des éléments majeurs de la dynamique fluviale, fournissant l'énergie au «moteur morphologique».

L'ambition d'un mode de gestion de certains barrages par des «lâchers morphogènes » est de permettre de préserver ou de restaurer, lorsque cela est possible et pertinent, certaines propriétés de la dynamique fluviale. De nombreux compartiments de l'hydrosystème peuvent être altérés par d'autres actions anthropiques (chenalisation, extractions de granulats, etc.) et les «lâchers morphogènes» restent des mesures de mitigation à l'origine de fonctionnalités écologiques encore atteignables dans un contexte plus ou moins fortement artificialisé (Fig. 2).

\section{Protocole de mise en œuvre de lâchers morphogènes}

La procédure d'élaboration d'un projet de lâcher morphogène en aval d'un barrage comprend sept étapes principales (Fig. 3) sous la forme d'un logigramme.

Ces différentes étapes doivent être menées en concertation étroite avec toutes les parties prenantes. Il est fondamental de noter ici que le passage aux étapes $2,3,5$, et 6 ne sera possible que si l'étape précédente a été partagée et validée par tous. De même, le bilan établi à l'étape 7 et le passage soit à une routine, soit à des itérations vers des étapes précédentes devra être partagé et validé par toutes les parties prenantes.

Le principe de mise en œuvre du logigramme est le suivant.

\section{1 Étape 1 : diagnostic d'état}

Un tel diagnostic de l'état hydromorphologique et écologique du tronçon concerné par le projet de lâchers morphogènes permet d'établir la trajectoire évolutive du cours d'eau, de comprendre sa dynamique fonctionnelle. Trois objectifs sont ainsi assignés à ce diagnostic d'état:

- identifier tous les dysfonctionnements hydromorphologiques et écologiques présents dans le tronçon concerné;

- évaluer quelle part des dysfonctionnements observés est imputable aux modifications hydrologiques et sédimentaires induites par le barrage;

- déterminer parmi les dysfonctionnements observés, et au regard du contexte hydrosédimentaire local, ceux qui pourraient être atténués, voire résorbés par des lâchers d'eau morphogènes (Fig. 4).
Il sera indispensable de décrire les indicateurs utilisés pour ce travail. Au terme de ce diagnostic d'état, celui-ci est partagé et validé par toutes les parties prenantes. Il peut être décidé par toutes les parties que les dysfonctionnements observés ne sont pas liés à la modification de l'hydrologie ou du transport solide induite par la présence du barrage et qu'aucune amélioration ne peut être attendue par la mise en œuvre de lâchers morphogènes. On sort alors de la procédure pour aller vers une étude de solutions techniques visant à remédier à ces dysfonctionnements.

\section{2 Étape 2 : détermination des objectifs et des indicateurs de réussite}

Le diagnostic d'état étant validé, il s'agit alors de voir quels sont les objectifs visés par le lâcher morphogène (ex.: décolmatage du substrat, arrachement des jeunes pousses de végétation alluviale, etc.). Par exemple, un objectif visé pourrait être de réduire à moins de $50 \%$ la superficie colmatée des radiers du cours d'eau cible. La figure 5 présente cet objectif et les résultats. Il est constaté une part de $80 \%$ pour les classes 3-4-5 avant le lâcher avec en haut les classes de colmatage utilisées et en bas, le résultat post-lâcher sur une station repère. Le résultat avec moins de $60 \%$ de superficie colmatée pour les classes 3-4-5 après le lâcher montre que l'objectif est presque atteint... Les objectifs sont, dès cette étape, quantifiés via des indicateurs et des seuils de réussite (ex. : on vise le décolmatage d'au moins $30 \%$ de la superficie $\mathrm{du}$ lit, le dessablement d'au moins $50 \%$ du tronçon, l'arrachement d'au moins $50 \%$ des jeunes pousses de l'année de salicacées dans la bande active, etc.). Le partage et la validation de ces indicateurs et ces seuils de réussite sont primordiaux car ce sont eux qui vont déterminer le dimensionnement «idéal» du lâcher réalisé lors de l'étape 3.

\section{3 Étape 3 : dimensionnement et solutions alternatives}

\subsection{1 Étape 3a: dimensionnement}

Il s'agit, pour la première mise en œuvre de cette étape 3 , de déterminer (par calculs simples, modélisations diverses, REX de cas similaires, etc.) le dimensionnement d'un lâcher «idéal » qui permettrait d'atteindre les objectifs fixés en 2. À ce stade, on ne tient compte d'aucune contrainte (ex. : pour atteindre les objectifs quantifiés fixés en 2, il faut $n \mathrm{~m}^{3} / \mathrm{s}$ pendant $n$ heures). Ce dimensionnement est généralement entaché d'une assez grande incertitude prédictive car ni les calculs ni même la modélisation numérique ou physique de processus tels que le transport solide ou l'arrachement des végétaux ne sont à ce jour fiables à $100 \%$. L'objectif est de fournir ici des ordres de grandeur des processus attendus.

\subsection{2 Étape 3b: étude de solutions alternatives}

Outre l'étude de dimensionnement de lâchers d'eau, on s'attachera à évaluer si des solutions alternatives peuvent éventuellement permettre d'atteindre les objectifs fixés en 2 ou sont nécessaires en complément de lâchers pour assurer leur réussite (ex.: un arrachage mécanique de la végétation alluviale, un pâturage des milieux riverains concernés par des animaux, etc.). 


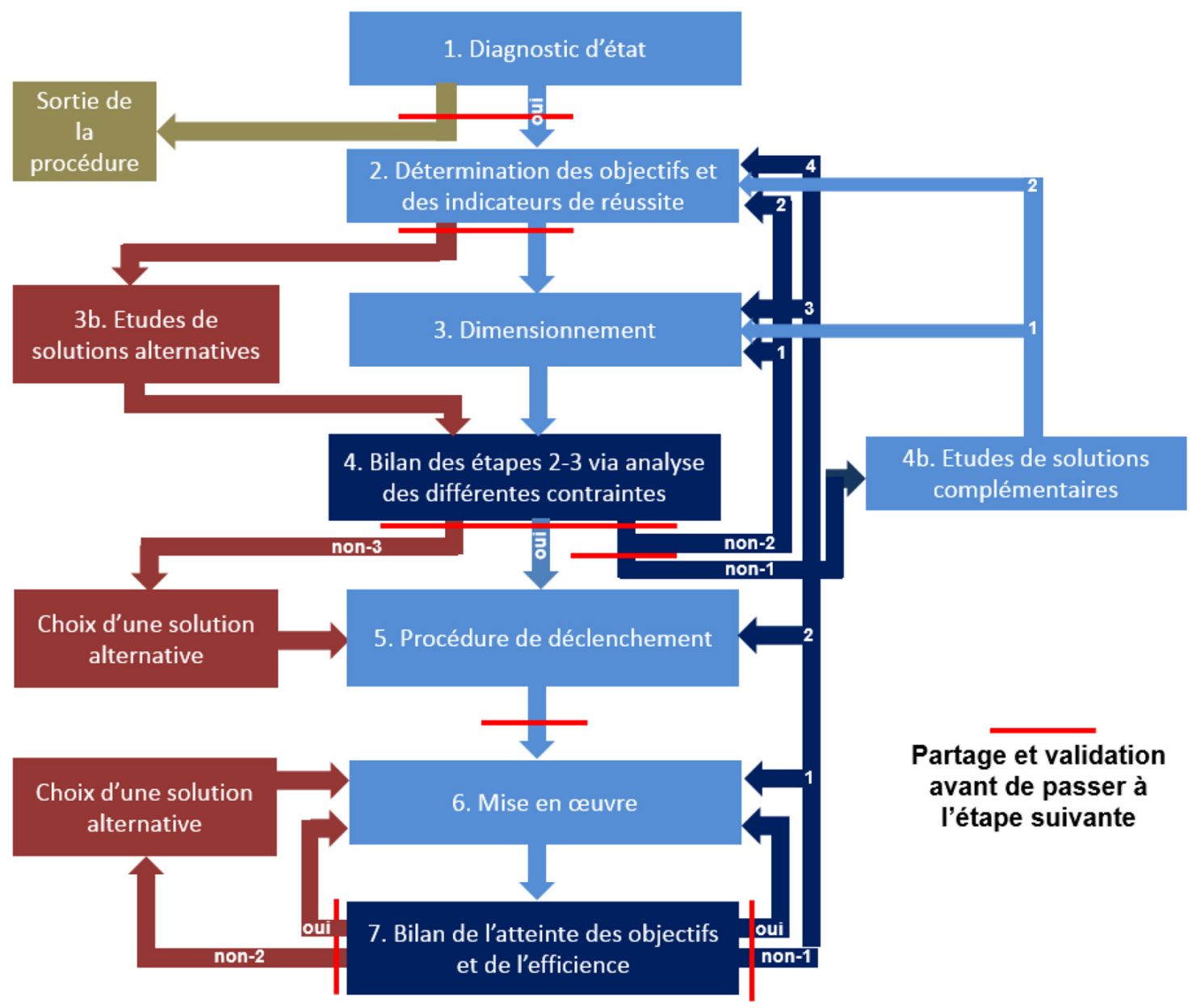

Fig. 3. Logigramme conceptuel des sept étapes d'élaboration d'un projet de lâchers morphogènes.
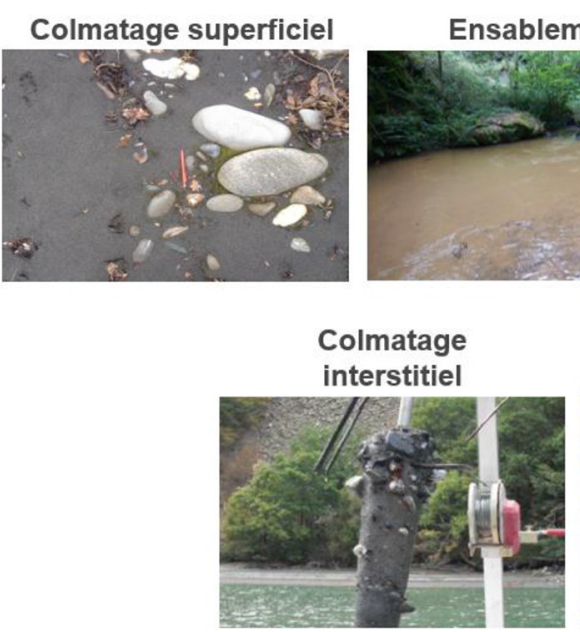

Ensablement

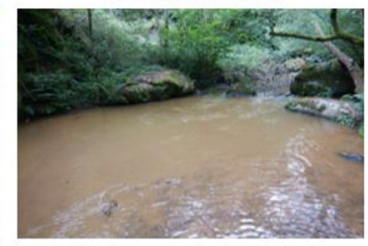

Surdéveloppement des macrophytes

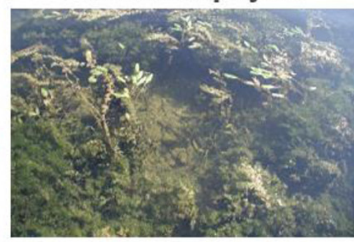

Surdéveloppement du périphyton

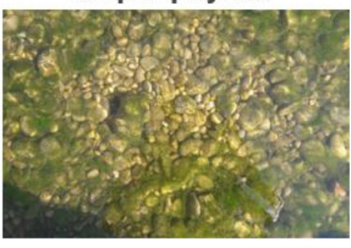

Surdéveloppement de la végétation dans le lit mineur

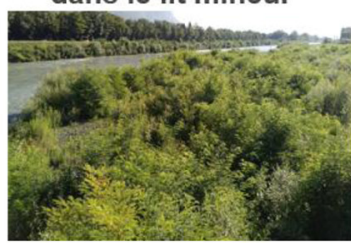

Fig. 4. Illustrations des principaux dysfonctionnements potentiellement atténuables, voire résorbables, par des lâchers morphogènes (EDF).

Il faut noter ici que les solutions alternatives ont pour défaut de n'être souvent que « sectorielles » (c'est-à-dire n'agissant que sur un seul compartiment) là où un lâcher peut être plus « intégrateur » (il peut par exemple à la fois arracher la végétation pionnière ET contribuer à la recharge sédimentaire par érosion latérale...).

\section{4 Étape 4 : bilan des étapes 2 et 3 via l'analyse des contraintes et des coûts}

C'est le premier des deux bilans de la procédure. Il vise notamment à valider ou non le dimensionnement déterminé en 

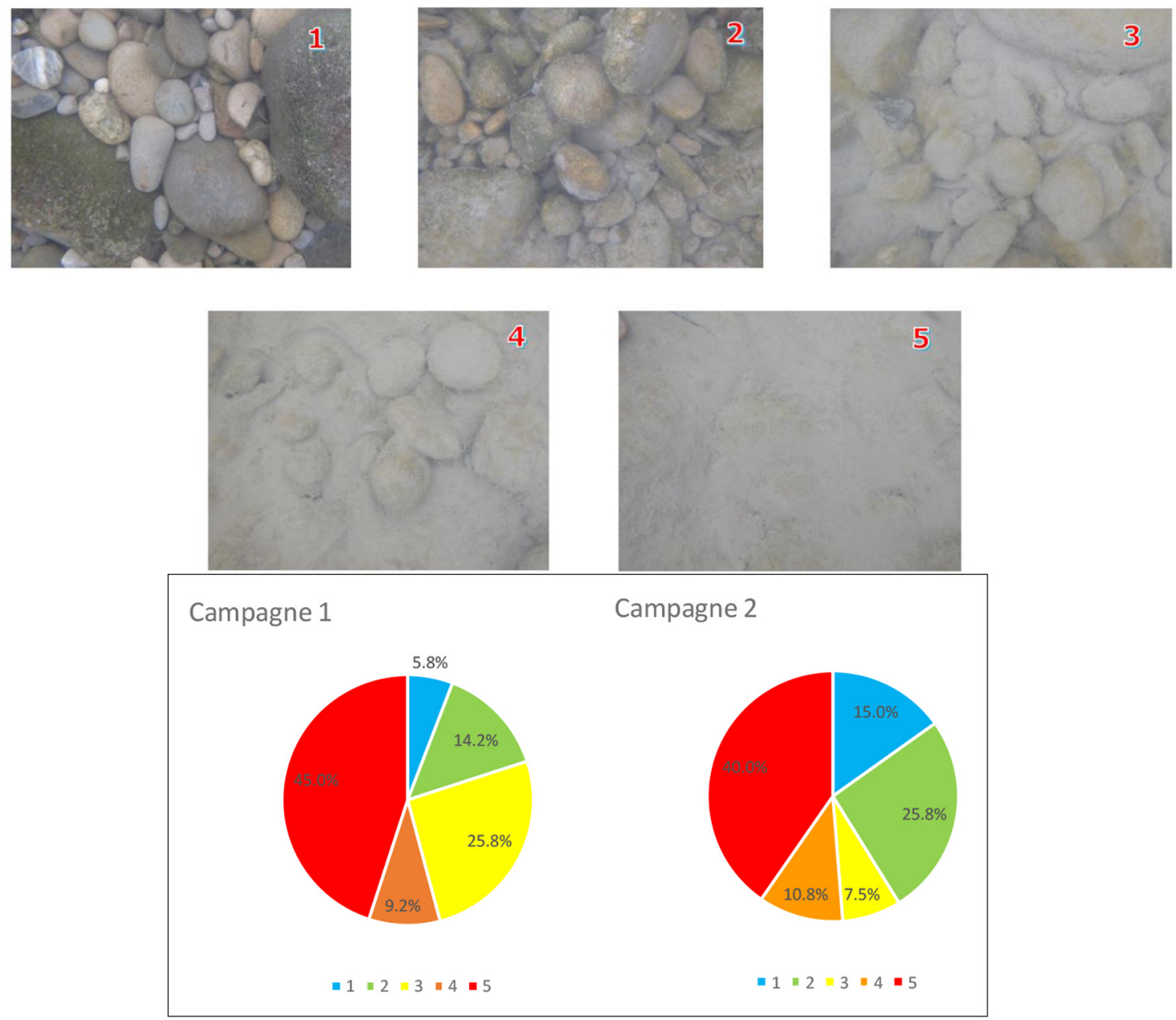

Fig. 5. Exemple d'objectif visé et de résultat avant et après lâcher.

3a ou éventuellement la solution alternative proposée en $3 \mathrm{~b}$ (ex. : il n'est pas possible d'atteindre le débit du lâcher « idéal » car la taille des vannes ne le permet pas, il n'est pas possible d'atteindre la durée du lâcher «idéal» car le volume du réservoir n'est pas suffisant, etc.). En plus des diverses contraintes techniques, sociétales, environnementales, de sécurité des personnes, etc. ce bilan permet aussi de vérifier le niveau d'efficience (rapport coût/efficacité) de l'opération de lâcher par rapport à une éventuelle solution alternative.

Au terme de ce bilan, quatre solutions sont envisageables. Si le lâcher n'est pas retenu, trois autres options doivent être alors évaluées (retour en $3 b$ ). Les itérations peuvent être réalisées plusieurs fois jusqu'à l'obtention d'une solution adaptée qui permette de passer à l'étape 5 .

Il y aura passage à l'étape 5 si le dimensionnement «idéal» est réalisable et si l'analyse de l'efficience du lâcher par rapport à des solutions alternatives est confirmée.

La première option à analyser (NON-1, Fig. 3), sauf cas particulier (voir NON-3) et de déterminer quelles mesures complémentaires au dimensionnement initial permettraient d'atteindre les objectifs fixés en étape 2 tout en intégrant les différentes contraintes (ex.: arrachage mécanique de la végétation sur certains sites où les débits seuls seraient insuffisants, Fig. 6). Il est alors possible de revenir au dimensionnement (3a) ou de remonter à l'étape 2 .

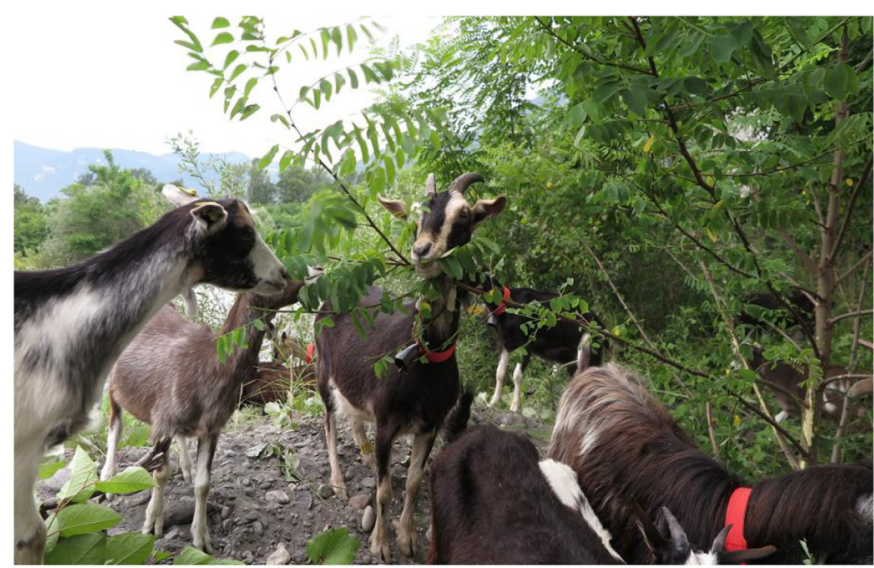

Fig. 6. Exemple de mesure alternative ou complémentaire. Pâturage caprin sur un banc végétalisé (EDF - Société d'économie alpestre de Savoie).

La seconde option (NON-2) conduit à déterminer si un autre dimensionnement, compte tenu des contraintes, permettrait néanmoins d'atteindre les objectifs fixés en 2 . Si ce n'est pas le cas, il conviendra de revenir à l'étape 2 et réviser à la baisse les objectifs puis à l'étape 3 pour redimensionner le lâcher avec ces nouveaux objectifs. 

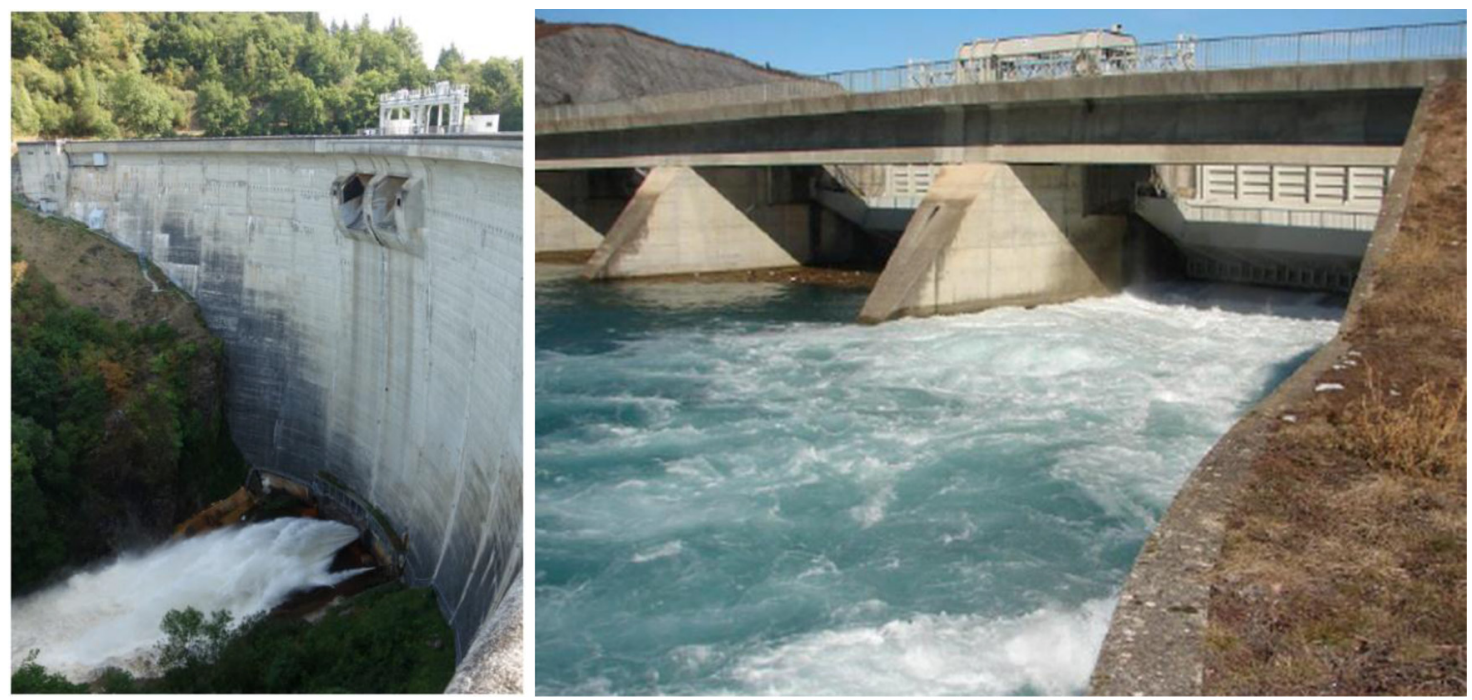

Fig. 7. Photographies de deux lâchers à vocation morphogène : (a) par la vanne de fond sur le barrage de Maury sur la Selves et (b) par les vannes de surface sur le barrage de la Saulce sur la Durance (EDF).

La troisième option (NON-3) conduit à envisager une solution alternative parmi celles recensées en $3 \mathrm{~b}$. Si cette option est retenue, les étapes 5, 6 et 7 sont réalisées sur la base de cette solution alternative, d'où la flèche qui part vers l'étape 5 . Cette solution alternative peut être proposée comme première option s'il est avéré que celle-ci présente des contraintes notablement plus faibles que la solution d'un lâcher (environnementale, de sécurité, financière, etc.). Cette décision doit être prise en étroite collaboration avec toutes les parties prenantes.

\section{5 Étape 5 : procédure de déclenchement de l'opération (un lâcher ou une solution alternative)}

Cette phase doit permettre de déterminer, sur la base de critères objectifs, à quel moment l'opération doit être mise en œuvre. Il s'agira principalement de critères quantitatifs directement associés aux dysfonctionnements observés (ex. : le déclenchement est réalisé si le niveau de colmatage moyen des radiers atteint par exemple 3 sur une échelle de 1 à 5 définie préalablement (ex. Fig. 5), si la superficie végétalisée par de jeunes pousses de salicacées atteint $30 \%$ du lit moyen, etc.). Cette étape repose sur la description d'un état initial, la détermination des critères de déclenchement et donc le choix des indicateurs et des seuils de déclenchement et leur réévaluation si nécessaire car il n'est pas rare que les critères de déclenchement soient réévalués au terme de l'étape 7.

\section{6 Étape 6 : mise en œuvre}

À ce stade de la procédure, on passe à la mise en œuvre de l'opération, qu'il s'agisse d'un lâcher (Fig. 7) ou d'une solution alternative (Fig. 6).

\section{7 Étape 7 : bilan de l'atteinte des objectifs et évaluation de l'efficience de l'opération}

Cette deuxième étape de bilan va permettre de faire le point sur la réussite de l'opération (ex. Fig. 8), via l'analyse des indicateurs de réussite.
Au terme de ce bilan, trois solutions sont envisageables : - le bilan est positif, les objectifs sont atteints avec une efficience acceptable. On passe alors à une «routine» de mise en œuvre récurrente basée sur les critères de déclenchement définis en étape 5 ;

- la mise en œuvre du lâcher (ou de la solution alternative) n'a pas permis d'atteindre les objectifs fixés en étape 2. On peut alors revenir à certaines étapes précédentes, depuis une nouvelle mise en œuvre (étape 6), si par exemple il est avéré que la première a été mal réalisée ou l'a été dans des conditions qui n'étaient pas optimales et qui expliquent la non-atteinte des objectifs, jusqu'à la redéfinition d'objectifs et d'indicateurs de réussite (étape 2). Le processus d'itération doit être limité en nombre, notamment pour ce qui concerne la mise en œuvre. Ce point doit être débattu avec les parties prenantes lors du bilan mais il est couramment admis que une à trois mises en œuvre expérimentales suffisent pour avoir une idée assez précise de l'efficience de l'opération;

- s'il n'est toujours pas possible d'atteindre les objectifs fixés, même révisés à la baisse, on retient une autre solution opérationnelle et à sa mise en œuvre.

\section{Conclusion}

Les lâchers morphogènes sont une solution technique potentiellement efficace pour améliorer le fonctionnement de rivières dont les forts débits sont contrôlés par des barrages en amont. Cette stratégie ne doit cependant être mise en œuvre qu'après un diagnostic d'état qui est complet (compartiments géomorphologiques et écologiques) et partagé, et qui doit évaluer si les dysfonctionnements observés sont bien liés à des modifications hydrologiques et si les lâchers morphogènes sont bien la solution la plus pertinente.

$\mathrm{Si}$ au terme de cette première étape de la procédure proposée ici, le choix de réaliser un ou plusieurs lâchers morphogènes est effectué, la mise en œuvre des étapes suivantes va permettre de définir des objectifs précis et 


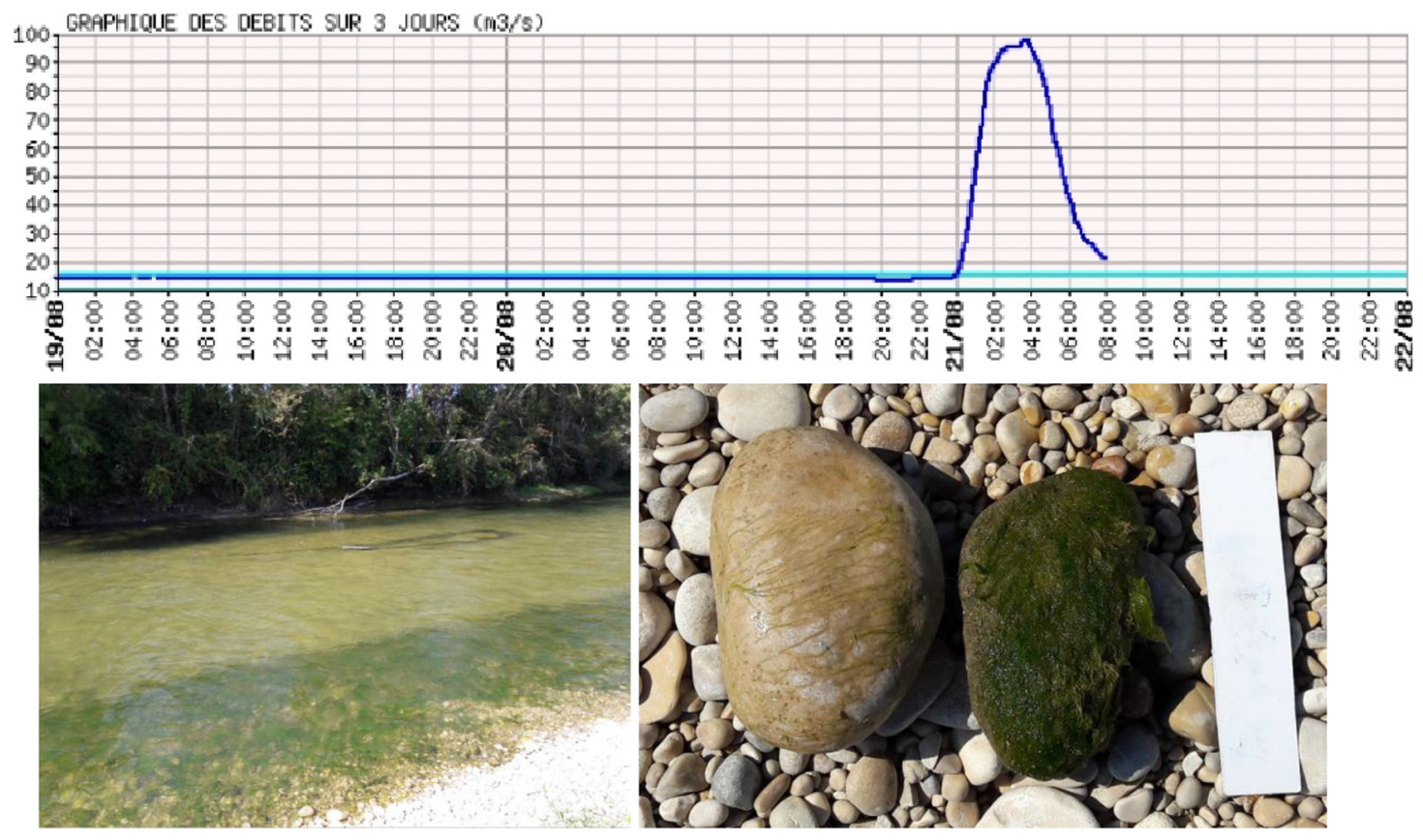

Fig. 8. Disparition du périphyton sur le cours d'eau cible suite à un lâcher de désalgage de $2-3 \mathrm{~h}$ à $100 \mathrm{~m}^{3} / \mathrm{s}$. On distingue bien la partie du chenal qui a été remobilisée et celle qui n'a pas bougé. À droite, deux galets de même taille prélevés dans les deux zones.

réalistes, de dimensionner les lâchers, de les mettre en œuvre et de suivre leur efficacité sur le court, moyen et long termes.

Il est important de noter que de nombreuses contraintes peuvent potentiellement contrecarrer les objectifs et dimensionnements initiaux. Elles peuvent même aller jusqu'à rendre un lâcher impossible. De plus, la procédure est très souvent itérative et cela a parfois, pour conséquence, une révision à la baisse des objectifs initiaux.

Il faut noter aussi l'importance du suivi scientifique de ces opérations, au moins les premières années, pour valider leur efficacité et l'intérêt de les poursuivre dans le temps.

Notons enfin que la compréhension des processus géomorphologiques et plus encore des relations entre ces processus et le fonctionnement écologique des cours d'eau est encore très largement du domaine de la recherche. L'approche itérative et le suivi de l'efficience des opérations peuvent participer à l'amélioration de nos connaissances dans ces domaines.

\section{Références}

Arthington AH, Zalucki JM. 1998. Comparative evaluation of environmental flow assessment techniques: review of methods. LWRRDC Occas Pap Ser 27/98: 141.

Batalla R, Vericat D, Tena A. 2014. The fluvial geomorphology of the lower Ebro (2002-2013): bridging gaps between management and research. Cuad Investig 40: 29-51. Available from https://doi.org/ 10.18172/cig.2569.

Benson MA, Thomas DM. 1966. A definition of dominant discharge. Int Assoc Sci Hydrol Bull 11(2): 76-80. Available from https://doi. org/10.1080/02626666609493460.

Brandt SA. 2000. Classification of geomorphological effects downstream of dams. Catena 40: 375-401. Available from https://doi.org/10.1016/S0341-8162(00)00093-X.
Camenen B, Grabowski RC, Latapie A, Paquier A, Solari L, Rodrigues S. 2015. On the estimation of the bed-material transport and budget along a river segment: application to the Middle Loire River, France. Aquat Sci 78: 71-81. Available from https://doi.org/ 10.1007/s00027-015-0442-3.

Helland-Hansen E, Milhous RT, Klingeman PC. 1974. Sediment transport at low shields parameters values. J Hydraul Div 100(1): 261-265.

Hjulström F. 1935. Studies of the morphological activity of rivers as illustrated by the River Fyris. Geol Inst Upsalsa 25: 221-527.

Klingeman PC, Bravard JP, Giuliani Y. 1994. Les impacts morphodynamiques sur un cours d'eau soumis à un aménagement hydroélectrique à dérivation : le Rhône en Chautagne (France). Rev Geogr Lyon 69(1): 73-87. Disponible sur https://doi.org/10.3406/ geoca.1994.4240.

Kondolf GM. 1997. Hungry water: effects of dams and gravel mining on river channels. Environ Manage 21: 533-551. Available from https://doi.org/10.1007/s002679900048.

Konrad CP, Olden JD, Lytle DA, Melis TS, Schmidt JC, Bray EN, Freeman MC, Gido KB, Hemphill NP, Kennard MJ, McMullen LE, Mims MC, Pyron M, Robinson CT, Williams JG. 2011. Large-scale flow experiments for managing river systems. Bioscience 61: 948 959. Available from https://doi.org/10.1525/bio.2011.61.12.5.

Loire R, Piégay H, Malavoi JR, Kondolf GM. Sous presse. From flushing flows to (eco)morphogenic releases: evolving terminology, practice, and integration into river management. Earth Sci Rev.

Olden JD, Konrad CP, Melis TS, Kennard MJ, Freeman MC, Mims MC, Bray EN, Gido KB, Hemphill NP, Lytle DA, McMullen LE, Pyron M, Robinson CT, Schmidt JC, Williams JG. 2014. Are largescale flow experiments informing the science and management of freshwater ecosystems? Front Ecol Environ 12: 176-185. Available from https://doi.org/10.1890/130076.

Milhous RT, Klingeman PC. 1973. Sediment transport system in a gravel-bottomed stream. In: Proc. 21st Ann. Speciality Conf. Hydraul. Div. A.S.C.E., 293-303. 
R.E. Loire et al. : La Houille Blanche 2020, 3, 66-75

Morel M, Tamisier V, Pella H. 2019. Revisiting the drivers of at-astation hydraulic geometry in stream reaches. Geomorphology 328: 44-56. Available from https://doi.org/10.1016/j.geo morph.2018.12.007.

Petts GE, Gurnell AM. 2005. Dams and geomorphology: research progress and future directions. Geomorphology 71: 27-47. Available from https://doi.org/10.1016/j.geo morph.2004.02.015.

Powell K. 2002. Open the floodgates. Nature 420: 356-358. Available from https://doi.org/10.1038/420356a.
Shields A. 1936. Application of similarity principles and turbulence research to bed-load movement, translated by W.P. Ott and J.C. Van Uchelen. Passadena, California.

Williams GP, Wolman MG. 1984. Downstream effects of dams on alluvial rivers. US Geol Surv Prof Pap (United States) J 1286: 83. Available from https://doi.org/10.1126/science.277.5322.9j.

Wolman MG, Leopold LB. 1957. River flood plains: some observations on their formation. U. S. Geol. Survey: Prof. Paper 282C: 87-109.

Wolman MG, Miller JP. 1960. Magnitude and frequency of forces in geomorphic processes. J Geol 68(1): 54-74.

Citation de l'article : Loire R, Piégay H, Malavoi J-R, Kondolf GM, Melun G. 2020. Les lâchers morphogènes depuis un barrage justification opérationnelle et protocole d'intervention. La Houille Blanche : 66-75 